\section{Effects of Trypsin and Thioglycollate upon the Nematocysts of the Sea Anemone}

AcL the hypotheses relating to the discharging mechanism of the stinging capsules of Coelenterates hitherto seem to postulate that an enhanced intracapsular pressure forces out the stinging thread. However, the elongated (about $50 \mu$ ) ellipsoid type of nematocysts ('penicilli' of Stephenson'), which can be isolated in quantity from the acontial filaments of Diadumene luciae in the form of a dense nematocyst suspension, have provided evidence that full hydrostatic pressure exists within the capsules in the resting state, so that only a releasing action from outside is necessary for the thread eversion to take place $^{2}$. At any rate, Glaser and Sparrow's view ${ }^{3}$ of the Metridium nematocyst as a simple osmometer cannot hold for the present material. The nematocysts of Diadumene possess a capsular wall impermeable to water, and will remain undischarged in distilled water, with their discharging capacity retained for several hours.

The commonest of discharge-evoking agents for the Diadumene nematocysts are acids and bases, both inorganic and organic. They act on the isolated nematocysts exactly in accord with the $p H$ values of the solution; $p \mathbf{H}$ limits to evoke discharge were determined to be $3 \cdot 0$ on the acid side, and $11 \cdot 0$ on the alkaline side. This result suggested a possibly protein nature of the hypothetical 'stopper' structure, which is situated at the distal end of the resting cap. sule to prevent the thread from being everted by the pressure from within. We have now obtained results which seem to support that view.

Merck trypsin in powder form was dissolved in distilled water buffered to $p \mathrm{H} 8 \cdot 3$ with sodium bicarbonate and applied to a population of isolated nematocysts, in concentrations of $1,0.3$ and 0.1 per cent. Discharge of practically 100 per cent of the nematocysts present took place. The effect was not an instantaneous one, but followed a regular sigmoid form of 'discharge percentage'-time curve, the level of 50 per cent discharge being reached in 2,3 and $7 \mathrm{~min}$. (temperature, $30^{\circ} \mathrm{C}$.), respectively, with the trypsin concentrations used. When unbuffered distilled water was used in preparing the enzyme solutions, the process was either stopped altogether by the low $p \mathrm{H}$ values (in 1 per cent solution, $p H 4 \cdot 3$ ), or considerably retarded (in 0.1 per cent solution, $p \mathrm{H} 4.8$ ). Cooling to $3^{\circ} \mathrm{C}$. practically stopped the process, which could be started again immediately on returning to room temperature. Heating the trypsin solution to $100^{\circ} \mathrm{C}$. for ten minutes deprived it of the effect irreversibly. No change in the appearances of the discharged nematocysts was found after having been immersed overnight in 1 per cent trypsin solution, $p H \quad 8 \cdot 3$.

A trial with $M / 4$ sodium thioglycollate solution $(p \mathrm{H} 8.4)$ seemed to us interesting in view of a brief report by Brown ${ }^{5}$ which suggested a keratin nature of the actinian nematocyst on the basis of detection of cystine and dissolution by alkaline sodium sulphide solution. The results we obtained were rather striking. A quite normal form of discharge was evoked at once in 100 per cent of the nematocysts on application of the thioglycollate solution, and it was followed by $a$ rapid and thorough dissolution of the capsule walls (in 5 min., see photographs). It is interesting to note that the discharged threads with their fine barbs did not dissolve, though they gave a visual impression of softening, curling, etc. Diluting the test solution to $M / 8$ rendered the discharging as well as the

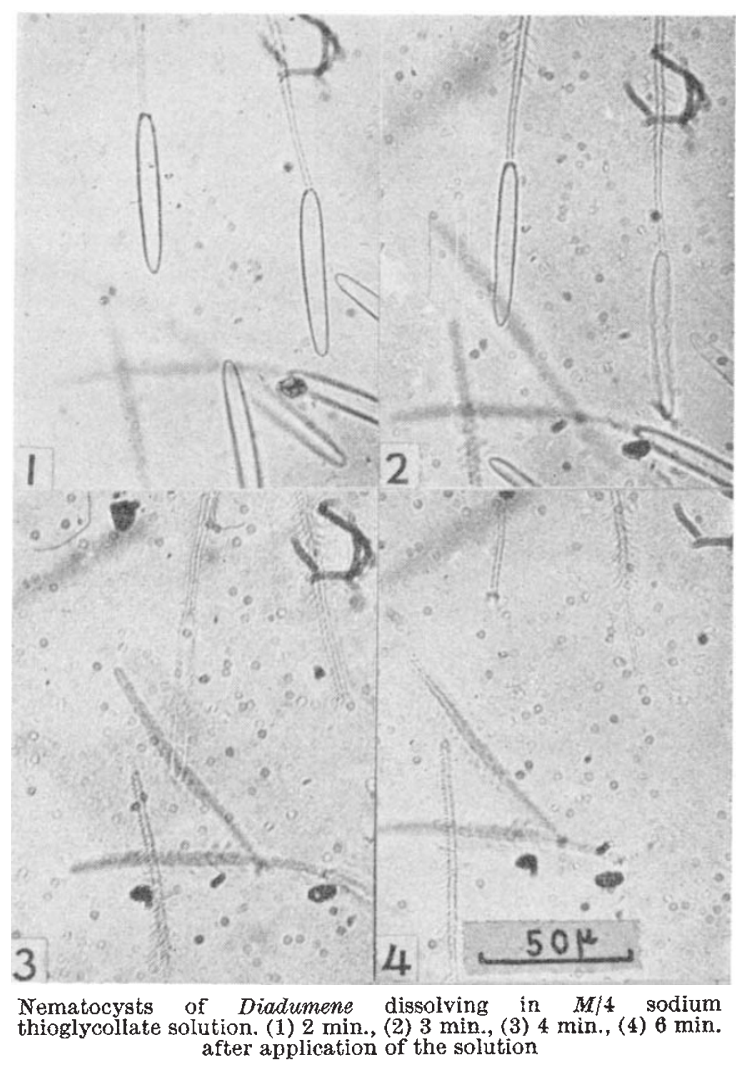

dissolving effect slower (discharge complete in $1 \mathrm{~min}$., dissolving in 15-20 min.). With further dilutions, there was no dissolution of the capsules, whereas the discharging process gave a sigmoid form of time course, 50 per cent discharge being reached in $7 \mathrm{~min}$. in $M / 20$, and in $20 \mathrm{~min}$. in $M / 40$ solution (tempera ture, $30^{\circ} \mathrm{C}$.). According to Goddard and Michaelis ${ }^{8}$, a $p \mathrm{H}$ above 10 is necessary to dissolve wool by thioglycollie acid. The present results may be remarkable for the much lower $p H$ values required for the dissolution of nematocysts.

It is true that some additional tests are still needed before the discharge-evoking effect of thioglycollate can be safely claimed as that of a keratin-reducing agent, for there are certain indications that any carboxylic acid anion may be able to evoke nematocyst discharge. If it is a specific effect of a disulphide reductant, our 'stopper' mechanism might consist of a mixed structure of keratin and non-keratin (some usual protein), a change in any of these components resulting in a loosening of the whole structure, and thus in the nematocyst discharge.

The actual mechanism within the acontial tissue by which, in response to an external stimulus, the 'stoppers' are released and the nematocysts are discharged is still in doubt.

\section{T. M. Yanagita \\ TUNEYO WADA}

Department of Biology, Ochanomizu University, Bunkyo Ward, Tokyo. Sept. 12.

' Stephenson, T. A., J. Mar. Biol. Assoc., 16, 173 (1929).

${ }^{2}$ Yanagita, T. M., J. Fac. Sci., Tokyo Imp. Univ., Sec. iv, 6, 97 (1943).

${ }^{3}$ Glaser, O. C., and Sparrow, C. M., J. Exp. Zool., 8, 361 (1909).

- Yanagita, T. M., and Wada, T., Nat. Sci. Rep., Ocha. Oniv., 4, 112 (1953).

s Brown, C. H., Nature, 166, 439 (1950).

${ }^{6}$ Goddard, D. R., and Michaelis, L., J. Biol. Chem., 106, 605 (1934). 